\title{
Anatomic Analysis of Blood Vessels in Germinal Matrix, Cerebral Cortex, and White Matter in Developing Infants
}

\author{
PRAVEEN BALLABH, ALEX BRAUN, AND MAIKEN NEDERGAARD \\ Departments of Pediatrics [P.B.], Pathology [A.B.], and Anatomy and Cell Biology [M.N.], New York \\ Medical College-Westchester Medical Center, Valhalla, NY 10595, U.S.A.; and Department of \\ Neurosurgery, Center for Aging and Developmental Biology, University of Rochester, Rochester, NY
}

14642 (M.N.)

\begin{abstract}
The germinal matrix (GM) located in the thick subependymal cell layer of the thalamostriate groove is a major site of cerebral hemorrhage in premature infants. Comparing the morphology of vasculature among GM, gray and white matter of the brain may help in understanding the pathogenesis of GM hemorrhage and also of periventricular leukomalacia. The objective of the present study was to determine the morphology of blood vessels in the GM, gray matter, and white matter and to examine maturational changes in the morphology of these vessels as a function of gestational age. We measured vessel density, percentage of blood vessel area, mean surface area, length, breadth, perimeter, radius, and shape of blood vessels in coronal sections of the GM, gray matter, and white matter in postmortem human brain samples for 17 fetuses and premature infants of gestational age 16-40 wk and 2 adults. We performed immunohistochemical staining using anti-laminin primary antibody, confocal microscopy to acquire images, and analysis using Metamorph version 6.1. Vessel den-
\end{abstract}

ABSTRACT

sity and the percentage of blood vessel area increased as a function of gestational age in the GM, gray matter, and white matter ( $p<0.001$ each). The blood vessel density and the percentage of blood vessel area were largest in the GM followed by gray matter and then white matter in all of the gestational age categories ( $p<0.001$ for all comparisons). Increased vascularity of the GM compared with gray and white matter may play a role in GM hemorrhage, whereas a relatively low vascularity of white matter may increase the propensity for the occurrence of periventricular leukomalacia in premature infants (Pediatr Res 56: 117-124, 2004)
GM, germinal matrix
Abbreviations
GMH, germinal matrix hemorrhage
IVH, intraventricular hemorrhage
PVL, periventricular leukomalacia

Germinal matrix hemorrhage (GMH)-intraventricular hemorrhage (IVH) continues to be an important cause of mortality and morbidity in premature infants $(1,2)$. The pathogenesis of IVH is not well understood. However, some theories concerning IVH include venous origin of hemorrhage (3), poorly developed tight junctions (4), greater lumenal areas (5), and low cerebral blood flow (6). Premature infants primarily bleed in the GM and not in gray and white matter, suggesting that there is an intrinsic weakness in the blood vessels of GM compared with blood vessels in other areas of the brain. Thus, this study is based on the premise that maturational changes in the morphology of GM blood vessels and difference in GM vasculature compared with gray and white matter may reveal

Received October 14, 2003; accepted March 24, 2004.

Correspondence: Praveen Ballabh, M.D., Pediatrics, NICU, 2nd floor, Main Building,

Westchester Medical Center, Valhalla, NY 10595, U.S.A.; e-mail: Pballabh@msn.com Supported by United Cerebral Palsy Educational and Research Foundation.

DOI: 10.1203/01.PDR.0000130472.30874.FF reasons for the vulnerability of the GM blood vessels to hemorrhage. In addition, development of white matter vasculature may be of importance with respect to the pathogenesis of periventricular leukomalacia (PVL), a white matter disease of premature newborns that is considered to be of hypoxicischemic origin (7).

The morphology of GM blood vessels has been studied by a number of investigators in beagle pups (8-10), rabbits (11), monkey fetuses (12), and premature human infants $(5,13)$. Studies on human infants have examined the pattern of vascularization (14), glial ensheathment of cerebral cortical blood vessels (15), and ultrastructure of GM vessels including luminal area (5) and endothelial area (5). However, a systematic study on human premature infants that examines vessel density, percentage of blood vessel area, and measurements of the blood vessel, including mean surface area, length, breadth, perimeter, radius, and shape of the blood vessels in the GM compared with gray and white matter, from early gestational age through term is lacking. 
We designed the present study to compare the morphology of blood vessels in the GM, gray matter, and white matter using immunohistochemistry and confocal microscopy. We also quantitatively examined changes in the morphology of brain vasculature in these areas from 16 to 40 wk gestation.

\section{METHODS}

Subjects. The Institutional Review Board at New York Medical College and Westchester Medical Center, Valhalla, NY, approved this study. The study material included autopsy brain samples from premature infants of postconceptional age 23-40 wk, from spontaneous abortuses of the gestational age 16-22 wk, and from adults. Gestational age was based on the last menstrual period of the mother. Only autopsy samples of $<12$-h postmortem interval were used for this study. All infants with major congenital anomalies, chromosomal defects, culture-proven sepsis, meningitis, or hypoxic-ischemic encephalopathy and infants who received extracorporeal membrane oxygenator treatment were excluded from the study. The characteristics of each subject included in this study are described in Table 1.

Human tissue collection and processing. Brain samples were obtained as coronal blocks by dissection of the following areas: GM from periventricular area in the region of thalamostriate groove and at the level of foramen of Monro, frontal white matter from the centrum semiovale, and frontal cortex (gray matter). All specimens were processed immediately after they were obtained. The samples were fixed in $4 \%$ paraformaldehyde in PBS (0.01 M, pH 7.4) for 18-24 h and then were cryoprotected by immersing into $20 \%$ sucrose in $0.01 \mathrm{M}$ PBS buffer for $48 \mathrm{~h}$. If the tissue did not sink, then 30 and $40 \%$ sucrose were used. Tissues were frozen after embedding them into OCT. Frozen coronal blocks were cut into $20-\mu \mathrm{m}$ sections using cryostat. For confirming GM, sections of tissues taken from the periventricular area of the caudate-thalamic groove were stained with hematoxylin and eosin and were examined with a light microscope.
The primary antibody used to stain the blood vessels was monoclonal mouse anti-human laminin antibody (catalog no. L8271, 1:500 dilution; Sigma Chemical, St. Louis, MO, U.S.A.). Secondary antibody was cy-5 conjugate goat antimouse (no. 115-175-146; Jackson Immunoresearch, West Grove, PA, U.S.A.). Before incubation with primary antibody, the sections were incubated with $5 \% \mathrm{NGS}, 1 \% \mathrm{BSA}$, and $0.2 \%$ Triton X-100 in PBS for $2 \mathrm{~h}$ at room temperature. The tissue sections were then incubated overnight at $4^{\circ} \mathrm{C}$ with the primary antibody diluted in PBS. After several washes in PBS, sections were incubated in secondary antibody for $2 \mathrm{~h}$ at room temperature. Finally, after washes in PBS, sections were mounted with Slow Fade Light Antifade reagent (Molecular Probes, Eugene, OR, U.S.A.) and were visualized under BIO-RAD confocal microscope (Richmond, CA, U.S.A.). Images were analyzed using Metamorph version 6.1 from Universal Imaging Corp. (Downingtown, PA, U.S.A.).

From each brain, a set of $8-10$ coronal sections taken as every 10th section were used for the study. From every section, five images were acquired from each area-GM, gray matter, and white matter-using $20 \times$ objective. Thus, a total of $120-150$ images $(8-10$ sections $\times 3$ areas $\times 5$ images $)$ per subject were acquired, and a total of $\sim 56,968$ blood vessel profiles in 2476 images were analyzed.Parameters studied were defined as follows:

Vessel density: number of blood vessels $/ \mathrm{mm}^{2}$

Percentage of blood vessel area: percentage of the area occupied by cross-section of all blood vessels in an image.

Mean surface area of the blood vessel: the average surface area of all blood vessels including the holes (lumen) present regardless of the hole filling in an image

Length: the span of the longest chord through the blood vessel

Breadth: the caliper width of the object perpendicular to the largest chord

Perimeter: distance around the edge of the blood vessel measuring from points of each pixel that defines the border

Table 1. Characteristics of the fetuses, infants, and adults

\begin{tabular}{|c|c|c|c|}
\hline Postconceptional age & Sex & Birth weight $(\mathrm{kg})$ & Cause of death/spontaneous termination \\
\hline $16 \mathrm{wk}$ & Male & & Cervical incompetence \\
\hline $18 w k$ & Female & & Cervical incompetence \\
\hline $20 w k$ & Female & & Preterm labor \\
\hline $21 \mathrm{wk}$ & Male & & Preterm labor \\
\hline $22 \mathrm{wk}$ & Female & 0.35 & Twins in preterm labor \\
\hline 23 wk & Female & 0.41 & Respiratory failure (died postnatal day 1$)$ \\
\hline $26 \mathrm{wk}$ & Male & 0.62 & Kidney failure (died postnatal day 3 ) \\
\hline $27 \mathrm{wk}$ & Female & 1.02 & Respiratory failure (died postnatal day 2) \\
\hline $28 \mathrm{wk}$ & Female, $2^{\text {nd }}$ twin & 0.55 & Respiratory failure (born $26 \mathrm{wk}$, died postnatal day 14 ) \\
\hline $30 \mathrm{wk}$ & Female & 1.34 & Pulmonary atresia (born $27 \mathrm{wk}$, died in operation room at $3 \mathrm{wk}$ of postnatal age) \\
\hline $32 \mathrm{wk}$ & Male & 1.4 & Perforated ileum (born $31 \mathrm{wk}$, died on postnatal day 5) \\
\hline $36 \mathrm{wk}$ & Male & 1.75 & After hernia repair (born $35 \mathrm{wk}$, died postnatal day 4) \\
\hline
\end{tabular}


Table 2. Dimensions of blood vessels in GM, gray matter, and white matter

\begin{tabular}{|c|c|c|c|c|c|}
\hline & 16-20 wk (4 subjects) & 21-23 wk (3 subjects) & 24-27 wk (4 subjects) & $28-32$ wk (3 subjects) & $36-40$ wk (3 subjects) \\
\hline \multicolumn{6}{|l|}{ GM } \\
\hline No. of BV/mm ${ }^{2 *}$ & $135.6 \pm 49.9$ & $157.1 \pm 59.1$ & $176.3 \pm 49.6$ & $203.2 \pm 64.9$ & - \\
\hline Total BV area $(\%)^{*}$ & $4.0 \pm 1.5$ & $6.3 \pm 1.4$ & $6.24 \pm 2.3$ & $7.6 \pm 1.8$ & - \\
\hline Length $(\mu)$ & $34.3 \pm 10.5$ & $40.0 \pm 12.2$ & $34.2 \pm 8.2$ & $36.5 \pm 9.7$ & - \\
\hline Breadth $(\mu)$ & $15.8 \pm 3.6$ & $18.3 \pm 4.3$ & $15.8 \pm 2.5$ & $16.6 \pm 2.9$ & - \\
\hline Perimeter $(\mu)$ & $101.5 \pm 35.6$ & $136.2 \pm 55.1$ & $103.1 \pm 34.4$ & $110.4 \pm 32.6$ & - \\
\hline \multicolumn{6}{|l|}{ Gray matter } \\
\hline No. of $\mathrm{BV} / \mathrm{mm}^{2 *}$ & $78.55 \pm 37.69$ & $103.8 \pm 39.9$ & $103.6 \pm 39.8$ & $111.5 \pm 31.5$ & $254.7 \pm 90.3$ \\
\hline Total BV area $(\%)^{*}$ & $2.8 \pm 1.5$ & $3.48 \pm 1.7$ & $3.7 \pm 1.3$ & $3.7 \pm 1.0$ & $5.4 \pm 1.3$ \\
\hline Mean area of BV $\left(\mu^{2}\right)$ & $418.2 \pm 312.1$ & $341.3 \pm 192.4$ & $393.41 \pm 221$ & $328.2 \pm 120.1$ & $224.6 \pm 128$ \\
\hline Length $(\mu)$ & $46.8 \pm 28.7$ & $38.9 \pm 13.5$ & $45.6 \pm 14.4$ & $42.4 \pm 10.6$ & $31.0 \pm 12.5$ \\
\hline Breadth $(\mu)$ & $15.5 \pm 5.5$ & $15.8 \pm 4.6$ & $15.2 \pm 3.7$ & $14.3 \pm 2.1$ & $14.1 \pm 9.8$ \\
\hline No. of $\mathrm{BV} / \mathrm{mm}^{2 *}$ & $60.5 \pm 25.4$ & $62.5 \pm 20.7$ & $54.7 \pm 18.8$ & $48.6 \pm 20.3$ & $91.3 \pm 22.4$ \\
\hline Total BV area $(\%)^{*}$ & $2.1 \pm 1.2$ & $2.44 \pm 0.9$ & $2.0 \pm 0.8$ & $1.96 \pm 1.2$ & $3.2 \pm 1.0$ \\
\hline Mean area of BV $\left(\mu^{2}\right)$ & $400.3 \pm 274.4$ & $381.6 \pm 198.0$ & $403.7 \pm 298.0$ & $490.9 \pm 655.3$ & $346.3 \pm 126$ \\
\hline Length $(\mu)$ & $38.4 \pm 16.5$ & $42.2 \pm 14.3$ & $41.3 \pm 17.1$ & $44.0 \pm 31.9$ & $40.3 \pm 10.6$ \\
\hline Breadth $(\mu)$ & $15.4 \pm 4.1$ & $16.2 \pm 4.1$ & $16.0 \pm 5.1$ & $17.9 \pm 14.4$ & $15.3 \pm 3.3$ \\
\hline Perimeter $(\mu)$ & $111.7 \pm 56.2$ & $148.8 \pm 59.3$ & $128.4 \pm 82.3$ & $121.2 \pm 69.2$ & $109.7 \pm 27$ \\
\hline Radius $(\mu)$ & $11.5 \pm 4.3$ & $12.4 \pm 3.76$ & $12.2 \pm 5.2$ & $11.9 \pm 4.5$ & $11.6 \pm 2.8$ \\
\hline Shape factor & $0.46 \pm 0.19$ & $0.35 \pm 0.15$ & $0.42 \pm 0.15$ & $0.42 \pm 0.1$ & $0.46 \pm 0.26$ \\
\hline
\end{tabular}

BV, blood vessels.

Data are mean \pm SD.

* $P<0.001$, an increase in blood vessels $/ \mathrm{mm}^{2}$ and total blood vessel area as a function of gestational age (ANOVA).

Mean radius: average distance from centroid to all points along the edge

Shape factor: the shape factor is equal to $4 \pi \mathrm{A} / \mathrm{P} 2$, where $\mathrm{P}$ is perimeter and $\mathrm{A}$ is area; a value of 0 indicates a flat object, and a value of 1 indicates a circle

Statistical analysis. Vessel density; percentage of blood vessel area; and dimensions of the blood vessel, including length, breadth, perimeter, radius, and shape, were studied as a function of gestational age, and these parameters were compared among GM, gray matter, and white matter. For assessing the effect of gestational age on blood vessel density and dimensions of the blood vessels in the GM, gray matter, and white matter, infants were stratified into five categories with respect to gestational age: $16-20,21-23,24-27,28-32$, and 36-40 wk. This stratification was based on viability and maturity considerations of fetuses and preterm infants. We used Kruskal-Wallis test (ANOVA model) to compare the parameters across the gestational age categories. Pairwise comparison was done for each of the variables vessel density, total blood vessel area, mean blood vessel area, length, breadth, perimeter, mean radius, and shape factor between GM versus gray matter, GM versus white matter, and gray matter versus white matter in all five gestational age categories using MannWhitney $U$ test. The pairwise comparison of characteristics of blood vessels between 36- to 40-wk newborns and adults was also done using Mann-Whitney $U$ test. A $p<0.05$ was considered significant.

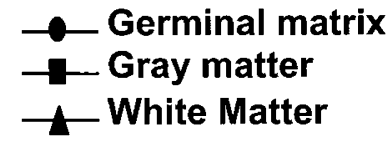

\section{2}

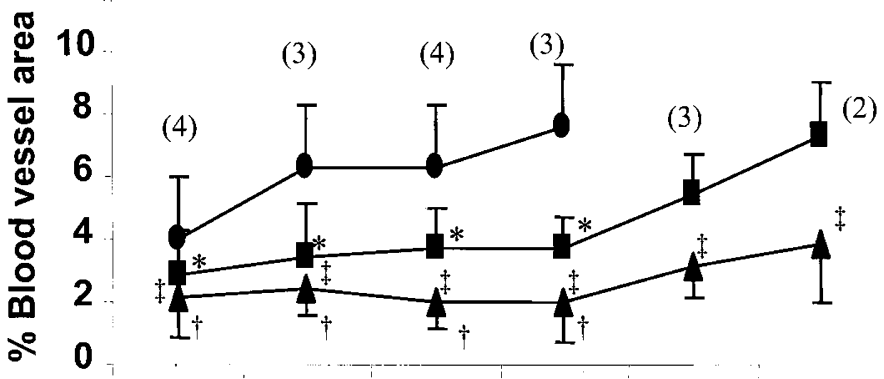

$\begin{array}{lllll}16-20 & 21-23 \quad 24-27 \quad 28-32 & 36-40 & \text { Adults }\end{array}$ Post-conceptional Age

Figure 1. Percent blood vessel area in $16-20,21-23,24-27,28-32$, and $36-40$ week subjects in germinal matrix, gray and white matter. Data are mean and standard deviation. Percent blood vessel area increased with increase in the gestational age of subjects in the GM, gray and white matter $(p<0.001$ each, ANOVA). Percent blood vessel area was greater in the GM compared to cortex and gray matter for all gestational age categories $(p<0.001$ each). In addition, percent blood vessel area in gray and white matter was increased in adults compared to mature newborns. The number in the parentheses indicates the number of subjects. ${ }^{*} p<0.001$ for the comparison between germinal matrix and gray matter. $\dagger p<0.001$ for the comparison between germinal matrix and white matter. $\ddagger p<0.001$ for the compariaon between gray and white matter. 


\section{RESULTS}

Characteristics of the subjects included in the study are depicted in Table 1. Autopsy brain material obtained from the GM, gray matter, and white matter in 19 subjects was studied. The subjects were divided into five gestational-age categories: 16-20 (four subjects), 21-23 (three subjects), 24-27 (four subjects), 28-32 (three subjects), and 36-40 (three subjects) wk. We also included two adult brains in this study.

Effect of gestational age on morphology of blood vessels. The effect of gestational age on percentage of blood vessel area in the GM, gray matter, and white matter is shown in Table 2 and Figures 1 and 2. The percentage of blood vessel area increased as a function of gestational age in the GM, gray matter, and white matter ( $p<0.001$ each, ANOVA model). Likewise, vessel density (number of blood vessels $/ \mathrm{mm}^{2}$ ) also increased as a function of gestational age in the GM, gray matter, and white matter ( $p<0.001$ each, ANOVA; Table 2, Figs. 2 and 3). The percentage of blood vessel area and the number of vessels in the GM increased from 16 to $32 \mathrm{wk}$ and in gray matter from 16 to $40 \mathrm{wk}$ of gestational age, whereas these measurements in white matter increased significantly only after 32 wk of gestational age. Dimensions of the blood

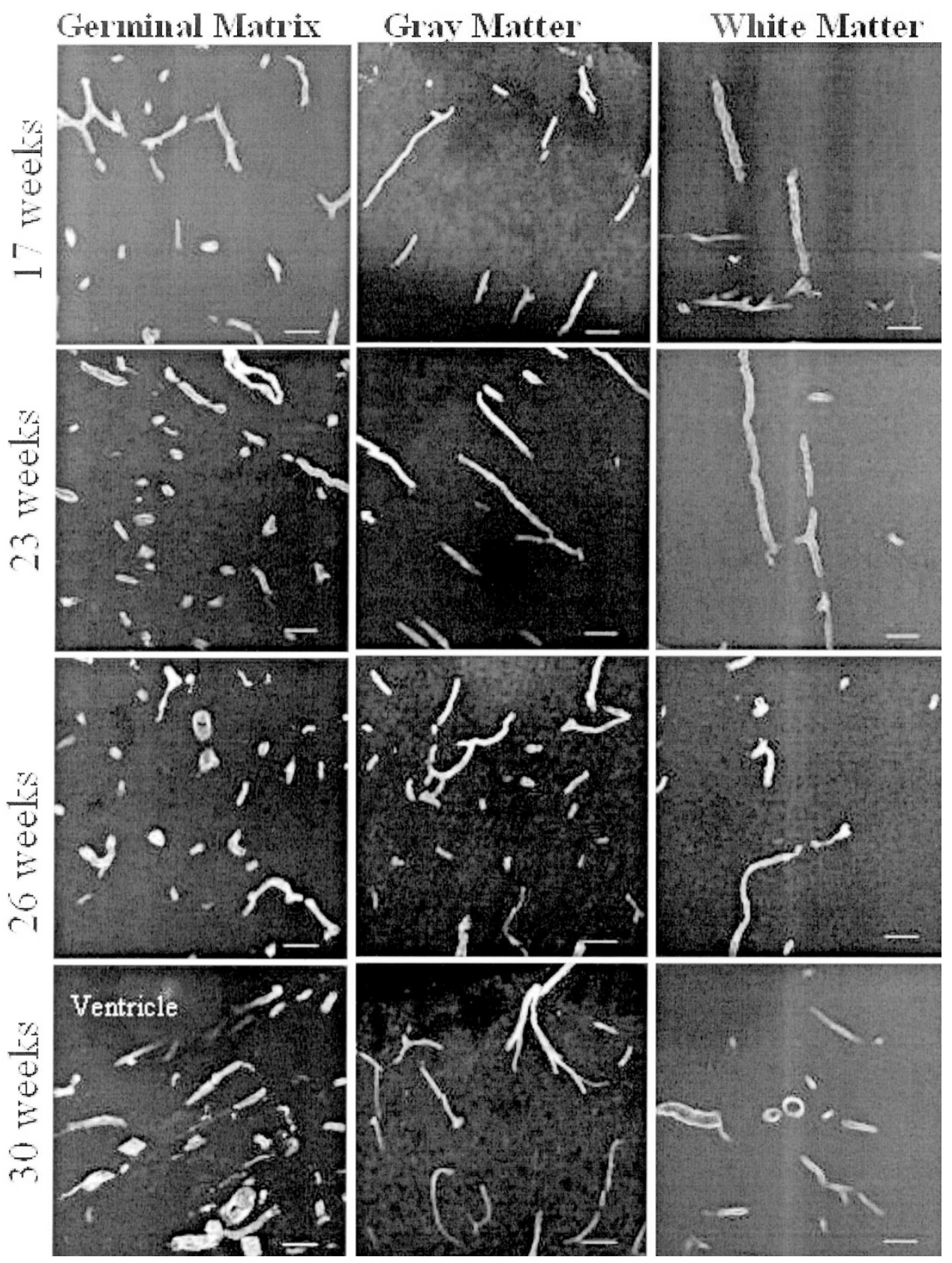

Figure 2. Coronal sections through germinal matrix, gray and white matter in 17, 23, 26, and 30 week subjects showing laminin immunohistochemical staining. Images of germinal matrix, gray and white matter are in left, middle and right column respectively and images of $17,23,26$, and 30 weeks are in rows starting from the top. The figure shows that number of vessels and percent blood vessel area are greater in germinal matrix followed by gray and then white matter. In addition, number of vessels and percent blood vesel are greater in higher gestational age subjects in GM, gray and white matter. 


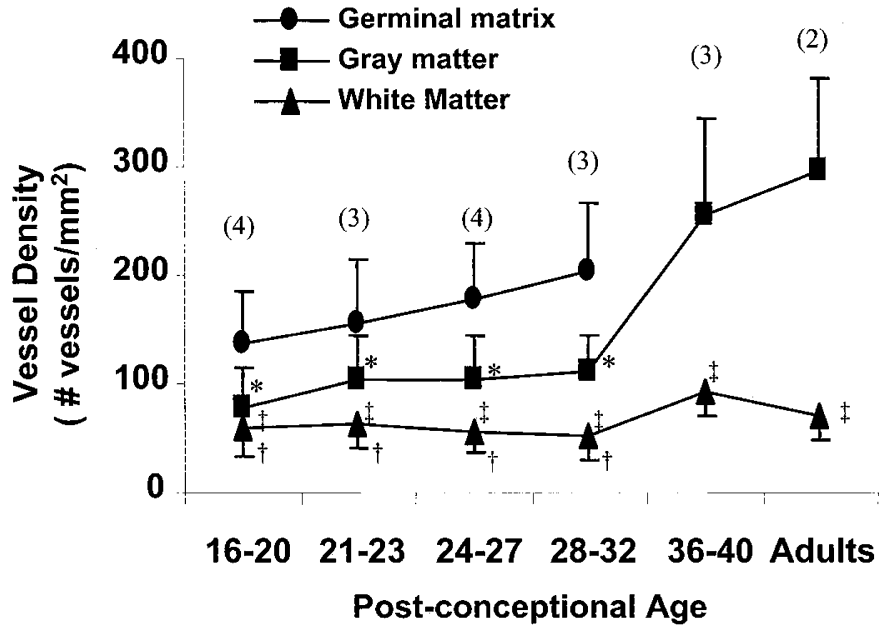

Figure 3. Blood vessel density in $16-20,21-23,24-27,28-32$, and $36-40$ week subjects in germinal matrix, gray and white matter. Data are mean and standard deviation. Blood vessel density increased in the GM, gray and white matter with increase in the gestational age of subjects $(p<0.001$ each, ANOVA). Blood vessel density was greater in the GM compared to cortex and gray matter for all gestational age categories $(p<0.001)$. In addition, blood vessel density in gray matter was increased in adults compared to mature newborns. However, blood vessel density in white matter was lower in adults compared to mature newborns. The number in parentheses indicates the number of subjects. ${ }^{*} p<0.001$ for the comparison between germinal matrix and gray matter. $\dagger p<0.001$ for the comparison between germinal matrix and white matter. $\ddagger p<0.001$ for the comparison between gray and white matter.

vessels in the GM, gray matter, and white matter, including mean surface area of blood vessels, length, breadth, perimeter, and radius in the gestational age categories, are depicted in Table 2. No consistent upward or downward trend in their values was noted with an increase in the gestational age.

Comparison among GM, gray matter, and white matter. The blood vessel density and the percentage of blood vessel area were the largest in the GM followed by gray matter and then white matter in all gestational age categories, and comparisons between them were statistically significant $(p<0.001$ for all; Tables 2 and 3). Shape factor for the blood vessels was significantly greater in the GM compared with gray matter for all gestational age categories, indicating that blood vessels were more circular in the GM than in gray matter. This comparison was also significant for GM versus white matter except for 16-20 and 28-32 wk gestational age. The comparison of mean surface area, length, breadth, radius, and perimeter of the GM blood vessels with gray and white matter did not show any consistent increase or decrease for the various gestational age categories. For 36- to 40-wk infants, we observed higher vessel density and greater percentage of vessel area in gray matter compared with white matter $(p<0.001$ each). However, mean area $(p<0.001)$, length $(p<0.001)$, breadth $(p<0.05)$, perimeter $(p<0.001)$, and radius $(p<$ 0.001 ) of blood vessels were significantly smaller in gray matter compared with white matter. We did not find significant GM in a 36-wk infant; thus, images from GM were not acquired on this infant.

Comparison between adult and 36- to 40-wk newborns. In close similarity to 36- to 40-wk newborns, gray matter of adult brains had increased vessel density with greater percentage
Table 3. $P$ values for the Comparisons Between Germinal Matrix (GM), Gray Matter (Cortex) and White Matter (WM)

\begin{tabular}{|c|c|c|c|}
\hline & GM vs Cortex & $\mathrm{GM} v s \mathrm{WM}$ & Cortex vs WM \\
\hline \multicolumn{4}{|l|}{$16-20 \mathrm{wk}$} \\
\hline No. of BV/mm² & $<0.001$ & $<0.001$ & $<0.001$ \\
\hline Total BV area (\%) & $<0.001$ & $<0.001$ & $<0.001$ \\
\hline Mean area of BV $\left(\mu^{2}\right)$ & $<0.001$ & NS & NS \\
\hline Length $(\mu)$ & $<0.001$ & $<0.05$ & $<0.01$ \\
\hline Breadth $(\mu)$ & $<0.05$ & NS & NS \\
\hline Perimeter $(\mu)$ & $<0.01$ & NS & $<0.05$ \\
\hline Radius $(\mu)$ & $<0.001$ & NS & $<0.05$ \\
\hline Shape factor & $<0.001$ & NS & $<0.01$ \\
\hline \multicolumn{4}{|l|}{$21-23$ wk } \\
\hline No. of $\mathrm{BV} / \mathrm{mm}^{2}$ & $<0.001$ & $<0.001$ & $<0.001$ \\
\hline Total BV area (\%) & $<0.001$ & $<0.001$ & $<0.003$ \\
\hline Mean area of BV $\left(\mu^{2}\right)$ & $<0.001$ & $<0.016$ & NS \\
\hline Length $(\mu)$ & NS & NS & NS \\
\hline Breadth $(\mu)$ & $<0.001$ & $<0.001$ & NS \\
\hline Perimeter $(\mu)$ & NS & NS & 0.02 \\
\hline Radius $(\mu)$ & NS & $<0.001$ & $<0.001$ \\
\hline Shape factor & $<0.05$ & $<0.02$ & NS \\
\hline \multicolumn{4}{|l|}{$24-27 \mathrm{wk}$} \\
\hline No. of $\mathrm{BV} / \mathrm{mm}^{2}$ & $<0.001$ & $<0.001$ & $<0.001$ \\
\hline Total BV area (\%) & $<0.001$ & $<0.001$ & $<0.01$ \\
\hline Mean area of BV $\left(\mu^{2}\right)$ & NS & NS & NS \\
\hline Length $(\mu)$ & $<0.001$ & $<0.01$ & $<0.01$ \\
\hline Breadth $(\mu)$ & $<0.05$ & $<0.05$ & NS \\
\hline Perimeter $(\mu)$ & $<0.001$ & $<0.05$ & NS \\
\hline Radius $(\mu)$ & $<0.001$ & $<0.01$ & $<0.05$ \\
\hline Shape factor & $<0.001$ & $<0.02$ & NS \\
\hline \multicolumn{4}{|l|}{$28-32 \mathrm{wk}$} \\
\hline No. of $\mathrm{BV} / \mathrm{mm}^{2}$ & $<0.001$ & $<0.001$ & $<0.001$ \\
\hline Total BV area (\%) & $<0.001$ & $<0.001$ & $<0.003$ \\
\hline Mean area of BV $\left(\mu^{2}\right)$ & $<0.01$ & NS & NS \\
\hline Length $(\mu)$ & $<0.001$ & NS & $<0.05$ \\
\hline Breadth $(\mu)$ & $<0.001$ & NS & $<0.001$ \\
\hline Perimeter $(\mu)$ & $<0.01$ & NS & NS \\
\hline Radius $(\mu)$ & $<0.01$ & NS & NS \\
\hline Shape factor & $<0.001$ & NS & $<0.01$ \\
\hline
\end{tabular}

Comparison between blood vessels of gray matter and white matter for newborns of 36-40 wk gestational age was significant $(p<0.01)$ for all eight variables except for the shape factor. WM, white matter; NS, not significant.

area of blood vessels and decreased mean area, length, breadth, perimeter, and radius of the blood vessels compared with adult white matter (Table 4, Fig. 4).

We compared the vasculature of gray matter and white matter between 36- to 40-wk newborns and adults. We observed greater blood vessel density $(294.3 \pm 82.3$ versus 254.7.3 \pm 90.3$)$ and a higher percentage of blood vessel area ( $7.25 \pm 1.7$ versus $5.4 \pm 1.3$ ) in adult gray matter compared with newborn gray matter. In addition, we found greater percentage of blood vessel area $(3.9 \pm 1.9$ versus $3.2 \pm 1.0)$ and smaller blood vessel density $(68.6 \pm 19.5$ versus $91.3 \pm$ 22.4) in adult white matter compared with newborn white matter. Shape factor was higher for newborn gray and white matter vasculature, indicating a relatively round shape of newborn blood vessels and a flat shape of adult blood vessels. We are not showing the $p$ values for these comparisons because there were only two subjects in the adult group.

\section{DISCUSSION}

We observed that blood vessel density and percentage of total blood vessel area increased with gestational age 16 through $32 \mathrm{wk}$ 
Table 4. Dimensions of BV in gray matter (cortex) and white matter in 36-40 wk newborns and adults

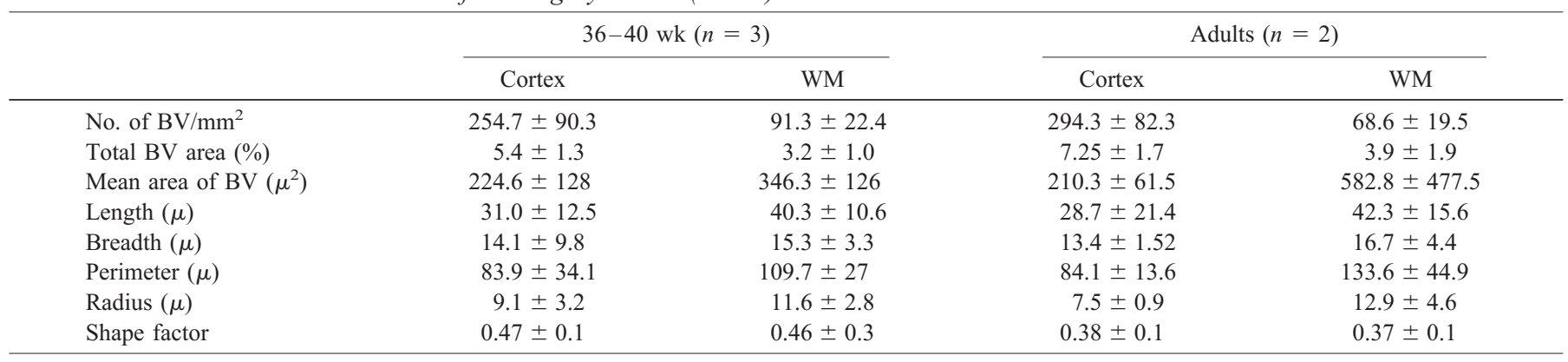

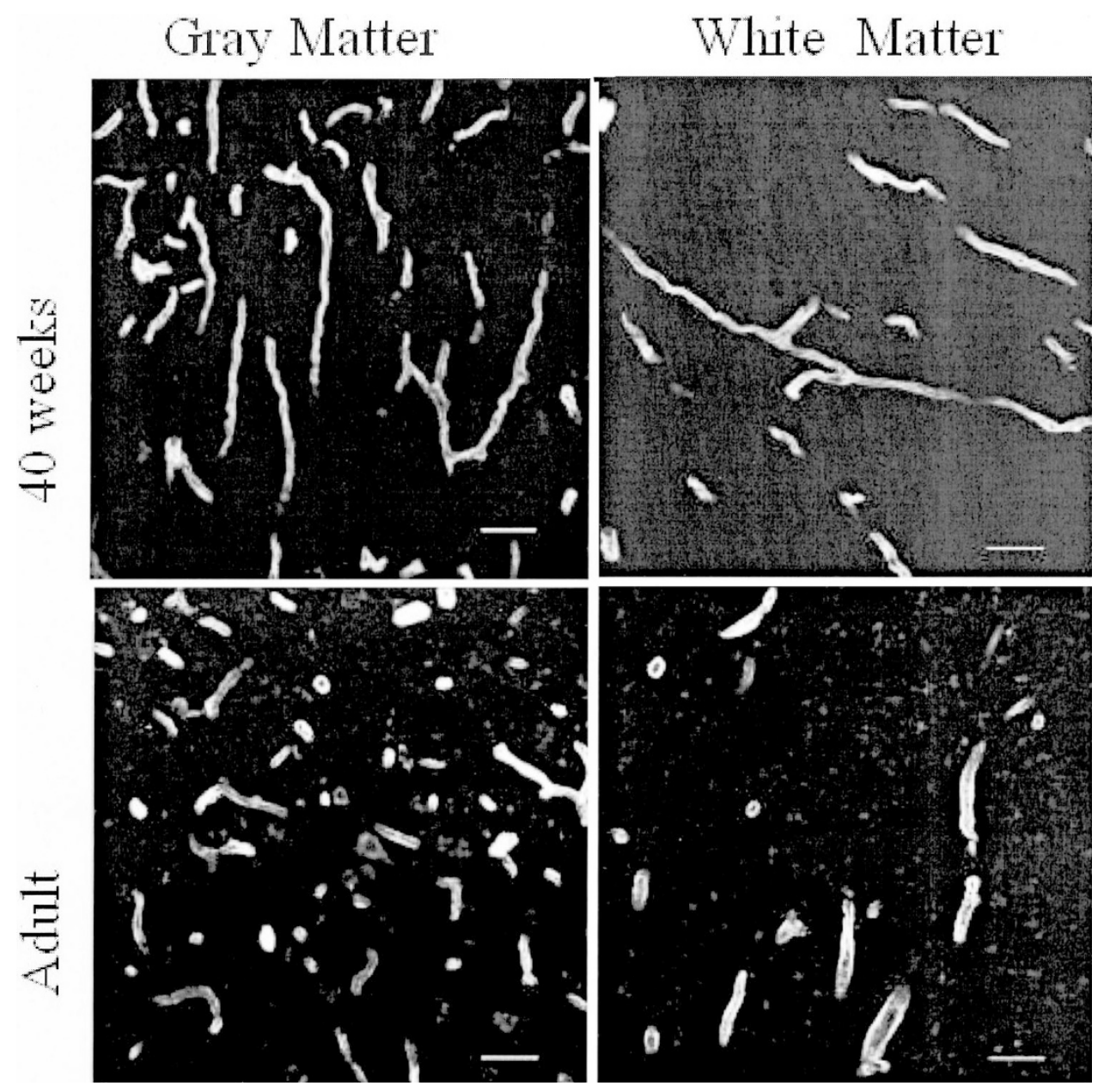

Figure 4. Coronal sections through gray and white matter in 40 week infant and adult showing laminin immunohistochemical staining. The figure shows that number of vessels and percent blood vessel area are greater in gray matter than white matter.

in the GM and 16 through $40 \mathrm{wk}$ in gray as well as white matter. In addition, blood vessel density and percentage of blood vessel area were largest in the GM followed by gray matter and then white matter in all gestational age categories.

We used monoclonal anti-laminin as a vascular marker to study the morphology of blood vessels because we observed consistent and uniform immunohistochemical staining with minimal background using this antibody in human brain tissues of all gestational ages, in addition to adults. However, limitations of our immunohistochemical method using laminin are that we were unable to measure the lumenal and endothelial area of blood vessels. In addition, this study could not differ- entiate among arteries, veins, and capillaries. Confocal microscopy was used to obtain two- and three-dimensional highresolution images of blood vessels in $20-\mu \mathrm{m}$ sections. We examined $8-10$ sections at an interval of $150-200 \mu \mathrm{m}$ in every subject for GM, white matter, and gray matter. Five images were acquired from each section, which gave us a large sample size from each subject.

Our finding of an increase in vessel density in the GM, gray matter, and white matter as a function of gestational age is supported by the previous study of Mito et al. (16). However, this study evaluated gray and white matter vasculature but not specifically blood vessels of the GM. They noted an increase in 
cortical vessel density at $26 \mathrm{wk}$ that peaked at $35 \mathrm{wk}$ but no significant difference in white matter blood vessels among subjects of various gestational ages. It is interesting that we observed an increase in blood vessel density and percentage of blood vessel area in gray matter from 16 to $40 \mathrm{wk}$ of gestational age, whereas these measurements in white matter did not increase until close to maturity $(36-40 \mathrm{wk})$. The description of phases in the development of human cerebral cortical vasculature also supports our finding of an increase in vascularization of cortex with gestational age (14). In addition, two to three times greater cerebral blood flow in term infants compared with preterm infants as measured by positron emission tomography indirectly supports our observation of an increase in cerebral vascularization as a function of gestational age (17).

Another key observation was that blood vessel density and the percentage of blood vessel area were largest in the GM followed by gray matter and then white matter for all gestational age categories. The finding of greater vascularity in the cortex compared with white matter is consistent with single photon emission tomography measurements that were made on premature infants and revealed four times greater cerebral blood flow in cortex than in white matter (18). However, to the best of our knowledge, there are no data in the literature on greater vascularity in the GM compared with other areas of the developing human brain. An ultrastructural study of the GM in premature beagle pups showed greater total vessel cross-sectional area in the GM compared with cortex (19), whereas another study on two normal newborn pups showed that the GM vessel density was similar to white matter and lower than that of cortex (20).

Notably, there was significantly less vascularity of white matter compared with the GM and gray matter for subjects of all gestational ages. In addition, there was no significant increase in the vascularity of white matter from 16 to $32 \mathrm{wk}$ of gestational age, and an increase in white matter vascularity was observed only after 32 wk. These observations are supported by the study demonstrating low cerebral blood flow in white matter compared with the basal ganglia and fronto-temporal cortex in premature infants (16). These findings are of particular importance in the context of PVL. PVL is a white matter disease of premature infants with a poorly understood cause. Our finding of relatively low white matter vascularity supports the notion that PVL consists of ischemic necrosis in the periventricular area as a result of decreased cerebral blood flow in critically ill premature newborns with hemodynamic instability.

It is interesting that in close similarity to newborns, adult gray matter had higher vessel density with greater percentage area of blood vessels and decreased mean area, length, breadth, perimeter, and radius of the blood vessels compared with adult white matter. These findings are supported by a recent study that measured dynamic cerebral perfusion in premature as well as mature neonates compared with that of adults using susceptibility contrast enhancement magnetic resonance imaging techniques (21). The investigators observed that images of relative cerebral blood flow from mature infants were comparable to adults, whereas relative cerebral blood flow data in premature infants showed perfusion voids predominantly in white matter and well-detected perfusion in gray matter. Thus, the resemblance of adult cerebral vasculature with that of mature but not premature newborns indicates that blood vessels in the gray and white matter of 36- to 40-wk newborns are approaching maturity.

The mean blood vessel, endothelial and lumen area have been studied in human GM and cerebral cortex by a previous investigator (5). This study does not provide information on the number of vessels (vessel density) and the percentage of the total blood vessel area in the GM, gray matter, and white matter. In contrast to the findings of this study, we did not observe larger mean blood vessel cross-sections in the GM compared with gray matter in 16-20 and 24-27 wk groups of subjects. Of note is that we did not observe a consistent change in the mean breadth of blood vessels in any area with increasing gestational age. Neither did we notice a consistent increase or decrease in the breadth of blood vessels comparing GM, gray matter, and white matter. However, studies on beagle pups showed slightly greater diameter of blood vessels in the GM compared with gray and white matter (9).

We found a higher shape factor for the GM blood vessels compared with gray matter in all subject groups and also in white matter of 21- to 27-wk subjects, meaning that GM blood vessels were more circular (less flat) in cross-section. We speculate that the more circular shape of blood vessels in cross-section indicates immaturity of vasculature in germinal matrix compared with other areas. This is indirectly based on evidence that during vascular development, as the pericytes and supporting cells associate with endothelial cells, the blood vessels become less dilated $(22,23)$.

The pathogenesis of GMH and PVL is currently not well understood. However, our finding of the greater vascularity of the GM compared with gray and white matter may explain the susceptibility of GM vasculature to hemorrhage. Likewise, our observation of a relatively low vascularity of white matter suggests that ischemia is likely to play a role in the pathogenesis of PVL. Additional research is needed to explain further how the different pattern of vascularity in GM and white matter contribute to the pathogenesis of GMH and PVL.

\section{REFERENCES}

1. Murphy BP, Inder TE, Rooks V, Taylor GA, Anderson NJ, Mogridge N, Horwood LJ, Volpe JJ 2002 Posthaemorrhagic ventricular dilatation in the premature infant: natural history and predictors of outcome. Arch Dis Child Fetal Neonatal Ed 87:F37-F41

2. Volpe JJ 1997 Brain injury in the premature infant. Neuropathology, clinical aspects, pathogenesis, and prevention. Clin Perinatol 24:567-587

3. Ghazi-Birry HS, Brown WR, Moody DM, Challa VR, Block SM, Reboussin DM 1997 Human germinal matrix: venous origin of hemorrhage and vascular characteristics. AJNR Am J Neuroradiol 18:219-229

4. Ment LR, Stewart WB, Ardito TA, Madri JA 1995 Germinal matrix microvascular maturation correlates inversely with the risk period for neonatal intraventricular hemorrhage. Brain Res Dev Brain Res 84:142-149

5. Grunnet ML 1989 Morphometry of blood vessels in the cortex and germinal plate of premature neonates. Pediatr Neurol 5:12-16

6. Meek JH, Tyszczuk L, Elwell CE, Wyatt JS 1999 Low cerebral blood flow is a risk factor for severe intraventricular haemorrhage. Arch Dis Child Fetal Neonatal Ed 81:F15-F18

7. Volpe JJ 2001 Neurobiology of periventricular leukomalacia in premature infants. Pediatr Res 50:553-562

8. Leuschen MP, Nelson RM Jr 1986 Telencephalic microvessels of premature beagle pups. Anat Rec 215:59-64

9. Trommer BL, Groothuis DR, Pasternak JF 1987 Quantitative analysis of cerebral vessels in the newborn puppy: the structure of germinal matrix vessels may predispose to hemorrhage. Pediatr Res 22:23-28

10. Ment LR, Stewart WB, Ardito TA, Madri JA 1995 Germinal matrix microvascular maturation correlates inversely with the risk period for neonatal intraventricular hemorrhage. Brain Res Dev Brain Res 84:142-149 
11. Sotrel A, Lorenzo AV 1989 Ultrastructure of blood vessels in the ganglionic eminence of premature rabbits with spontaneous germinal matrix hemorrhages. J Neuropathol Exp Neurol 48:462-482

12. Lenn NJ, Whitmore L 1985 Gestational changes in the germinal matrix of the norma rhesus monkey fetus. Pediatr Res 19:130-135

13. Povlishock JT, Martinez AJ, Moossy J 1977 The fine structure of blood vessels of the telencephalic germinal matrix in the human fetus. Am J Anat 149:439-452

14. Norman MG, O'Kusky JR 1986 The growth and development of microvasculature in human cerebral cortex. J Neuropathol Exp Neurol 45:222-232

15. Virgintino D, Monaghan P, Robertson D, Errede M, Bertossi M, Ambrosi G, Roncal L 1997 An immunohistochemical and morphometric study on astrocytes and microvasculature in the human cerebral cortex. Histochem J 29:655-660

16. Mito T, Konomi H, Houdou S, Takashima S 1991 Immunohistochemical study of vasculature in the developing brain. Pediatr Neurol 7:18-22

17. Altman DI, Powers WJ, Perlman JM, Herscovitch P, Volpe SL, Volpe JJ 1988 Cerebral blood flow requirement for brain viability in newborn infants is lower than in adults. Ann Neurol 24:218-26
18. Borch K, Greisen G 1998 Blood flow distribution in the normal human preterm brain. Pediatr Res 43:28-33

19. Leuschen MP, Nelson RM 1986 Telencephalic microvessels in premature beagle pups. Anat Rec 215:59-64

20. Trommer BL, Groothuis DR, Pasternak JF 1987 Qualitative analysis of cerebral vessels in newborn puppy: the structure of germinal matrix vessels may predispose to hemorrhage. Pediatr Res 22:23-28

21. Tanner SF, Cornette L, Ramenghi LA, Miall LS, Ridgway JP, Smith MA, Levene MI 2003 Cerebral perfusion in infants and neonates: preliminary results obtained using dynamic susceptibility contrast enhanced magnetic resonance imaging. Arch Dis Child Fetal Neonatal Ed 88:F525-F530

22. Hellstrom M, Gerhardt H, Kalen M, Li X, Eriksson U, Wolburg H, Betsholtz C 2001 Lack of pericytes leads to endothelial hyperplasia and abnormal vascular morphogenesis. J Cell Biol 153:543-553

23. Yancopoulos GD, Davis S, Gale NW, Rudge JS, Wiegand SJ, Holash J 2000 Vascular-specific growth factors and blood vessel formation. Nature 407:242-248 